\title{
Maneuverable Warhead Tracking Based on CKF
}

\author{
Zhimin Xiong a, Dangwei Wang ${ }^{\mathrm{b}}$ and Xiao Yan ${ }^{\mathrm{c}}$ \\ No. three department, Air Force Early Warning Academy, Wu Han, China; \\ a397862505@qq.com, bwdwjane@tom.com, clymaxioayan @126.com
}

\begin{abstract}
The performance of contemporary weapon systems has been enhanced by the development of military high-tech, which makes the maneuverable warhead as a new challenge of ballistic midcourse tracking. Aiming to solve the maneuvering warhead tracking, nonlinear filter algorithm, which is specially designed to track target with time-changing state and measurement models, is proposed. Recently the cubature Kalman filter (CKF) has been proposed to estimate the nonlinear system, providing more accurate state estimation. In this paper, the midcourse maneuvering warhead movement and measurement models are established. Then the cubature Kalman filter is presented to track the maneuvering warhead. The simulation results demonstrate the improved performance of CKF over traditional Kalman filter, which shows the shorter execution time of the former algorithm than the latter and the faster rate of convergence.
\end{abstract}

Keywords: Maneuverable Warhead; Cubature Kalman Filter; Ballistic Tracking.

\section{Introduction}

Ballistic missile has been the world most violent and destructive weapons in modern battle field. Early detection and pre-warning can improve the possibility of precision interception, which has always aroused widely attention of major military powers. The flight of ballistic missile can be divided into three phases: the boost phase, the midcourse ballistic phase and the reentry phase. As the main interception stage, the midcourse ballistic phase shows certain characteristics like long-time interception and relatively stable ballistic trajectory. However, the presence of maneuverable warhead has become the highlight in the target tracking field. The movement of Ballistic target is obvious nonlinear because of the effects from gravity, which makes the target motion state and radar measurements are non-linear changes state. Several of maneuvering targets tracking algorithms are developed, such as extended Kalman filter (EKF), unscented Kalman filter (UKF), or particle filter (PF) [1-3]. The extended Kalman filter is developed to solve the nonlinear filtering problems, based on applying traditional Kalman filter to the linearized models which has been linearized by Taylor expansion [4]. However, it suffers from widespread drawback that may cause serious filter divergence. Then the unscented Kalman filter has been introduced as a modified alternative to EKF for nonlinear state estimation [3]. The UKF has prominent performance in dealing with nonlinear problems, whose updating operation is realized by designing a few sigma points and calculating the propagation of these sigma points via non-linear functions. It simplifies the process of calculation and lessens the filter divergence [3]. The idea of the particle filter is to compute the conditional probability distribution function consists of a finite set of random particles and corresponding weights, which solves the integrals appearing in the filtering problem by means of stochastic Monte Carlo integration [5]. However, it has the inherent drawbacks like samples less of diversity [6].Lately, Arasaratnam and Haykin invented the cubature Kalman filter (CKF) [7, 8]. The CKF is to convert nonlinear filter into the calculation problem of the product between the nonlinear function and the Gaussian probability density function, based on a third degree spherical-radial cubature rule in the nonlinear Bayesian filter. The CKF performs better than the EKF and UKF [9].

The paper is organized as follows. The maneuvering midcourse ballistic warhead motion and measurement models are described first, and then cubature Kalman filter is used for ballistic missile tracking. The comparison of the CKF with the traditional tracking algorithms has been done by simulations and analysis in this paper shows that the new algorithm provides better estimation accuracy with minimal computational effort. 


\section{Mathematic Model of Multi-rotor}

\subsection{Movement Model}

Sometime after the launch of ballistic missile warhead flight separation from projectile, the target enters the middle phase of flight. Because the long distance between the radar and the warheads, and the Earth is actually elliptical shape, added second-order harmonic coefficients of the cue ball factor in the acceleration of gravity model based on the standard ellipsoid Earth gravity model, can achieve precise description of warhead motion model [10].

In order to use ellipsoid model for the Earth's gravity midcourse warhead trajectory modeling, the Earth-centered coordinate system $\mathrm{O}_{\mathrm{E}}-\mathrm{X}_{\mathrm{F}} \mathrm{Y}_{\mathrm{F}} \mathrm{Z}_{\mathrm{F}}$ is established, which has its origin at the Earth center ${ }^{O_{E}}$, its axes ${ }^{Z_{F}}$, and fundamental plane ${ }^{O_{E}} X_{F} Z_{F}$ coincident with the equatorial plane. Its axe ${ }^{O_{E} X_{F}}$ and ${ }^{O_{E} Y_{F}}$, however, rotate with the Earth around the Earth's spin axis $\mathrm{O}_{\mathrm{E}} \mathrm{Z}_{\mathrm{F}}$ as $\mathrm{O}_{\mathrm{E}} \mathrm{X}_{\mathrm{F}}$ points to the prime meridian. $L$ is the radar earth longitude, $B$ is the radar earth latitude; if $T$ is the set target warhead.

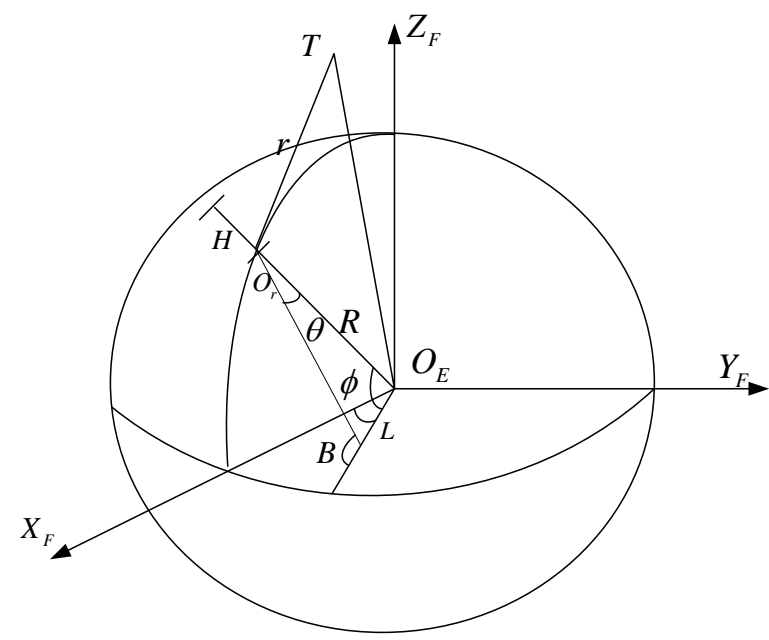

Fig 1.Ballistic Trajectory comparison

The forces act on the warhead $T$ can be expressed as

$a_{E}=a_{G}+a_{T}$

Where ${ }^{a_{G}}$ and ${ }^{a_{T}}$ denote the accelerations induced by gravity and drag, respectively. Throughout this

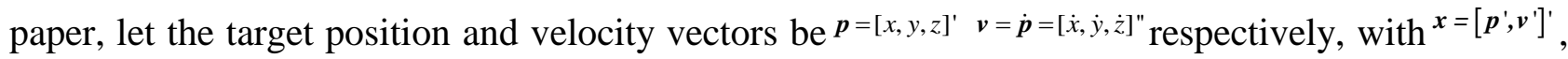
of the state-space models of a ballistic target has the form

$$
\dot{x}=\left[\begin{array}{ll}
v & a
\end{array}\right]^{\prime}
$$

Thus, the target acceleration model under EC coordinates can be expressed as [11] :

$$
\begin{aligned}
& a_{G}=\left[\begin{array}{l}
\ddot{x} \\
\ddot{y} \\
\ddot{z}
\end{array}\right]=-\frac{\mu}{p^{3}}\left[\begin{array}{l}
\left(1+\left(c_{e} / p^{3}\right)\left(1-5\left(z / p^{3}\right)^{2}\right)\right) x \\
\left(1+\left(c_{e} / p^{3}\right)\left(3-5\left(z / p^{3}\right)^{2}\right)\right) y \\
\left(1+\left(c_{e} / p^{3}\right)\left(1-5\left(z / p^{3}\right)^{2}\right)\right) z
\end{array}\right] \\
& p=\sqrt{x^{2}+y^{2}+z^{2}} \\
& c_{e}=3 J_{2} R^{2} / 2
\end{aligned}
$$

$\mu=3.986005 \mathrm{e} 14$ is the Earth gravitational constant, $J_{2}=0.108627989 \times 10^{-2}$ is the shape of the Earth dynamics factor, $R=6378137 m$ is the Earth radius.

Maneuvering of midcourse ballistic missile is produced by the thrust made by the rocket rooster built-in within a short time, which changes the acceleration of the warhead and the trajectory. The acceleration of the thrust is decided by the thrust-vectored engines T, the vertical direction $\boldsymbol{u}_{r}$ and the missile mass ratio $m$, as

$\boldsymbol{a}_{T}=T \boldsymbol{u}_{T} / \mathrm{m}$

And the missile mass ratio reduces as the fuel combustion:

$T=(d m / d t) g I_{s p}$ 
$(d m / d t)$ Is the missile fuel consumption per second, $g=\mu / R^{2}, I_{s p}$ is the specific impose.

We add a small increment to the azimuth angle and elevation angle of the velocity to make the thrust vector, the azimuth and elevation angle are:

$$
\begin{aligned}
& \alpha_{v}=\arctan (\dot{y} / \dot{x}) \\
& \lambda_{v}=\arctan \left(\dot{z} /\left(\sqrt{\dot{x}^{2}+\dot{y}^{2}}\right)\right)
\end{aligned}
$$

If the small increment of the azimuth angle and elevation angle are $\Delta \alpha$ and $\Delta \lambda$, so the azimuth angle and elevation angle of the thrust vector are:

$$
\begin{aligned}
& \alpha_{T}=\arctan (\dot{y} / \dot{x})+\Delta \alpha \\
& \lambda_{T}=\arctan \left(\dot{z} /\left(\sqrt{\dot{x}^{2}+\dot{y}^{2}}\right)\right)+\Delta \lambda
\end{aligned}
$$

So the thrust movement model can be expressed as:

$$
u_{T}=\frac{d m}{d t} \frac{g I_{s p}}{m}\left[\begin{array}{l}
\cos \left(\lambda_{T}\right) \cos (\arctan (\dot{y} / \dot{x})+\Delta \alpha) \\
\cos \left(\lambda_{T}\right) \sin (\arctan (\dot{y} / \dot{x})+\Delta \alpha) \\
\sin \left(\lambda_{T}\right)
\end{array}\right]
$$

Furthermore, the midcourse maneuvering warhead movement model can be expressed as [12]:

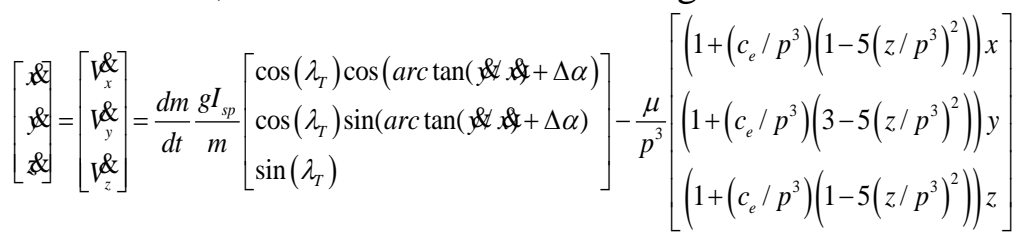

\subsection{Measurement Model}

In the formula (13) is provided on the basis of the operating state variables middle warhead $X=[x, y, z, \dot{x}, \dot{y}, \dot{z}]^{T}$, the missile in the ENU coordinate system state equation can be expressed as [13]:

$$
\frac{d X}{d t}=[\dot{x}, \dot{y}, \dot{z}, \ddot{x}, \ddot{y}, \ddot{z}]^{T}
$$

Discretization of the formula to obtain:

$$
X_{k+1 \mid k}=X_{k}+f\left(X_{k k}, t_{k}\right) \Delta t+F\left(X_{k k}\right) f\left(X_{k k}, t_{k}\right) \Delta t^{2} / 2
$$

Where $\Delta t$ the sampling interval determined by the tracking data rate, $F\left(X_{k \mid k}\right)$ is the Jacobi matrix as $f\left(X_{k \mid k}, k\right)$ relative to $X_{k \mid k}$ :

$$
F\left(X_{k \mid k}\right)=\left.\frac{d f}{d X}\right|_{X=X_{k \mid k}}=\left[\begin{array}{cc}
O_{3 \times 3} & I_{3 \times 3} \\
\frac{\partial \dot{r}}{\partial r} & -2 \omega \phi
\end{array}\right]_{X_{k \mid k}}
$$

The nonlinear measurement equation form[13] is then

$$
Z(k)=h\left(X_{k}\right)+\omega(k)=\left[\begin{array}{c}
\sqrt{x_{k}^{2}+y_{k}^{2}+z_{k}^{2}} \\
\tan ^{-1}\left(y_{k} / x_{k}\right) \\
\tan ^{-1}\left(z_{k} /\left(\sqrt{x_{k}^{2}+y_{k}^{2}}\right)\right)
\end{array}\right]+\left[\begin{array}{c}
n_{k}^{R} \\
n_{k}^{g} \\
n_{k}^{\theta}
\end{array}\right]
$$

Where $\omega(k)=\left[n_{k}^{R}, n_{k}^{\varphi}, n_{k}^{\theta}\right]^{T}$ is the measurement noise vector, $n_{k}^{R}, n_{k}^{\varphi}$ and $n_{k}^{E}$ are independent process and measurement Gaussian noise with zero means and variances $\sigma_{R}^{2}, \sigma_{\varphi}^{2}, \sigma_{\theta}^{2}$. Therefore, the measurement noise covariance matrix can be expressed as:

$$
R_{k}=\left[\begin{array}{ccc}
\sigma_{R}^{2} & 0 & 0 \\
0 & \sigma_{\varphi}^{2} & 0 \\
0 & 0 & \sigma_{\theta}^{2}
\end{array}\right]
$$

The Jacobi array $H\left(X_{k \mid k}\right)$ can be expressed as: 


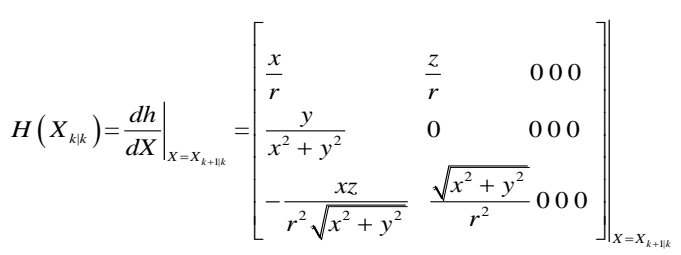

The nonlinear function $f(\sqcap, h(1)$ and the expression of their Jacobian matrix $F$ and $H$ have been determined; can use filtering technology to CKF mixed terminal ballistic target coordinates to track.

\section{A Target tracking based on CKF algorithm}

\subsection{Spherical-radial rule}

Middle warhead tracking is a typical nonlinear filtering problem in the process of target tracking. The gut of the CKF is to find a set of points and weights to compute the mean and covariance of the first two moments of the state in numeral. The CKF find the points and weights based on the third-degree spherical-radical rule.

The target motion equation of state can be expressed as:

$$
\begin{aligned}
& X_{k+1}=f_{k}\left(X_{k}\right)+v_{k} \\
& Z_{k+1}=h_{k+1}\left(X_{k+1}\right)+w_{k+1}
\end{aligned}
$$

Where $X_{k}$ is the state of the dynamic system, $f_{k}(\square) h_{k+1}(\square)$ are some known functions; $v_{k}$ and $w_{k+1}$ are independent process and measurement Gaussian noise with zeros means and covariances $Q_{k}$ and $R_{k}$, respectively.

Assume at time $k$ that the3posterior density of the state vector $p\left(X_{k} \mid Z^{k}\right)$ is known, the Bayesian filter in accordance with the following two steps:

(1)Time update

$$
p\left(X_{k} \mid Z^{k}\right)=\int p\left(X_{k+1} \mid X_{k}\right) p\left(X_{k} \mid Z^{k}\right) d X_{k}
$$

(2)Measurement update

$$
p\left(X_{k+1} \mid Z^{k+1}\right)=p\left(Z_{k+1} \mid X_{k+1}\right) p\left(X_{k+1} \mid Z^{k}\right) /\left(p\left(Z_{k+1} \mid Z^{k}\right)\right)
$$

Among them $p\left(Z_{k+1} \mid Z^{k}\right)$ normalizing factor as:

$$
p\left(Z_{k+1} \mid Z^{k}\right)=\int p\left(Z_{k+1} \mid X_{k+1}\right) p\left(X_{k+1} \mid Z^{k}\right) d X_{k+1}
$$

\subsection{Cubature Kalman Filter}

According to the Bayesian filter theory, the integrands of nonlinear filter are all of the form nonlinear plus Gaussian density. Aim to any arbitrary function $f(x)$; consider a multi-dimensional weighted integral of form,

$$
I(f)=\int_{R^{n_{x}}} f(x) \mathrm{N}(x ; \mu, P) d x \approx \sum_{i=1}^{2 n_{i}} \omega_{i} f\left(\mu+S \chi_{i}\right)
$$

$R^{n_{x}}$ is the region of the integration, $\mathrm{N}$ is the Gaussian distribution, $n_{x}$ is the state vector dimension. $\chi_{i}$ is the volume of points, $\omega_{i}=1 / 2 n_{x}$ is the volume point weights and $P=S S^{T}$ is covariance matrix for the Cholesky decomposition factors, namely, the volume of which point to set:

$$
\sqrt{n}\left\{\left[\begin{array}{l}
1 \\
0 \\
\vdots \\
0
\end{array}\right]\left[\begin{array}{l}
0 \\
1 \\
\vdots \\
0
\end{array}\right] \ldots\left[\begin{array}{l}
0 \\
0 \\
\vdots \\
1
\end{array}\right]\left[\begin{array}{l}
-1 \\
0 \\
\vdots \\
0
\end{array}\right]\left[\begin{array}{l}
0 \\
-1 \\
\vdots \\
0
\end{array}\right] \ldots\left[\begin{array}{l}
0 \\
0 \\
\vdots \\
-1
\end{array}\right]\right\}
$$

CKF can be summarized as follows:

Step1: CKF time update, factorize the covariance $P_{k k}$ and evaluate the cubature points:

$$
\left\{\begin{array}{l}
P_{k \mid k}=S_{k} S_{k}^{T} \\
\xi_{l, k+1}=S_{k} \cdot \chi_{l}+\hat{X}_{k}, l=1, \cdots, 2 n_{x}
\end{array}\right.
$$

Evaluate the propagated cubature points according to the state transition function $f_{k+1}(\square)$

$$
\xi_{l, k+1 \mid k}=f\left(\xi_{l, k+1}\right)=f\left(S_{k} \cdot \chi_{l}+\hat{X}_{k}\right), l=1, \cdots, 2 n_{x}
$$


Estimate the predicted state and the predicted error covariance

$$
\begin{aligned}
& \hat{X}_{k+1 \mid k}=\sum_{i=1}^{2 n_{x}} \omega_{i} \xi_{l, k+1 \mid k} \\
& P_{k+1 \mid k}=Q_{k+1}+\sum_{i=1}^{2 n_{k}} \omega_{i} \xi_{l, k+1 \mid k} \xi_{l, k+1 \mid k}^{T}-\hat{X}_{k+1 \mid k} \hat{X}_{k+1 \mid k}^{T}
\end{aligned}
$$

Step 2: CKF measurement update, factorize the covariance $P_{k \mid k}$ and evaluate the cubature points:

$$
\left\{\begin{array}{l}
P_{k+1 \mid k}=S_{k+1 k} S_{k+1 \mid k}^{T} \\
\xi_{l, k+1 \mid k}=S_{k+1 \mid k} \cdot \chi_{l}+\hat{X}_{k+1 k}, l=1, \cdots, 2 n_{x}
\end{array}\right.
$$

Evaluate the propagated cubature points according to the state transition function $h_{k+1}(\square)$

$$
\psi_{l, k+1 k}=h\left(\xi_{l, k+1 k k}^{\prime}\right)=h\left(S_{k+1 \mid k} \cdot \chi_{l}+\hat{X}_{k+1 \mid k}\right), l=1, \cdots, 2 n_{x}
$$

Estimate the predicted measurement and the innovation error covariance matrix

$$
\begin{aligned}
& \hat{Z}_{k+1 \mid k}=\sum_{l=1}^{2 n_{k}} \omega_{l} \psi_{l, k+1 \mid k} \\
& P_{Z, Z}=R_{k+1}+\sum_{l=1}^{2 n_{k}} \omega_{l}\left(\psi_{l, k+1 \mid k}-\hat{Z}_{k+1 \mid k}\right)\left(\psi_{l, k+1 \mid k}-\hat{Z}_{k+1 \mid k}\right)^{T}
\end{aligned}
$$

Estimate the CKF gain $K_{k+1}$

$$
K_{k+1}=P_{X, Z} P_{Z, Z}^{-1}
$$

Estimate cross- covariance matrix

$$
P_{X, Z}=\sum_{l=1}^{2 n_{k}} \omega_{l}\left(\xi_{l, k+1 \mid k}^{\prime}-\hat{X}_{k+1 \mid k}\right)\left(\psi_{l, k+1 \mid k}-\hat{Z}_{k+1 \mid k}\right)^{T}
$$

Estimate the update state and the corresponding error covariance

$$
\begin{aligned}
& \hat{X}_{k+1 \mid k+1}=\hat{X}_{k+1 \mid k}+K_{k+1}\left(Z_{k+1}-\hat{Z}_{k+1 \mid k}\right) \\
& P_{k+1 \mid k+1}=P_{k+1 \mid k}-K_{k+1} P_{Z, Z} K_{k+1}^{T}
\end{aligned}
$$

\section{Simulation and Analysis}

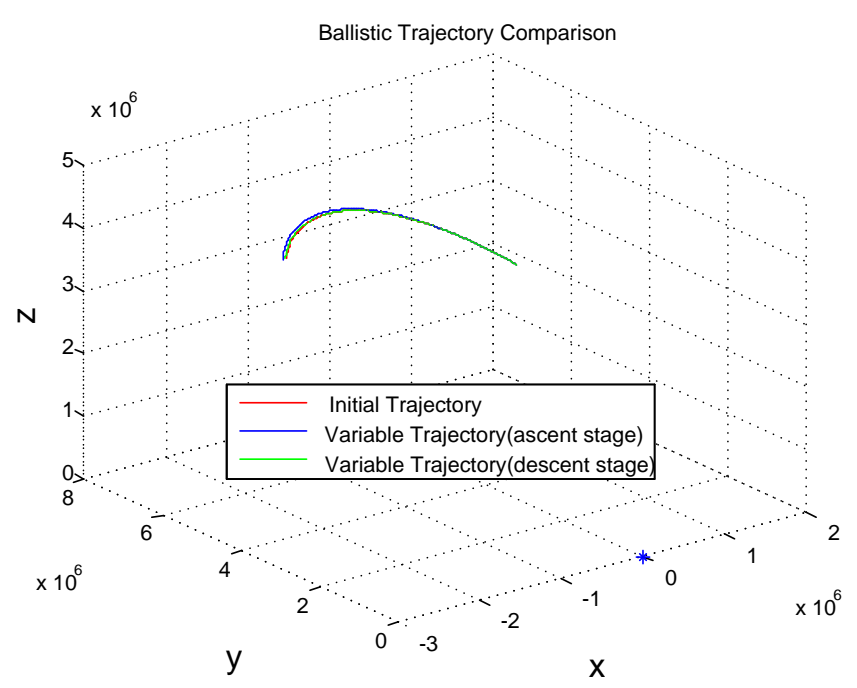

Fig 2.Ballistic Trajectory comparison

The trajectory for a maneuvering midcourse warhead is shown in Fig.2. The position, velocity and ballistic coefficient of a typical target trajectory are shown. The parameter of this typical target are: initial longitude130.4, initial altitude 43.5, initial height $166 \mathrm{~km}$, initial velocity $6.8 \mathrm{~km} / \mathrm{s}$, slant angle 43.3 ,azimuth angle 248.7 , the missile mass ratio $2300 \mathrm{~kg}$. the missile fuel consumption per second $50 \mathrm{~kg} / \mathrm{s}$, the specific impose $50 \mathrm{~s}$, length of maneuvering time $10 \mathrm{~s}$ radar's data rate is $10 \mathrm{~Hz}$, $\Delta \alpha=0.02 \mathrm{rad}, \Delta \lambda=0.5 \mathrm{rad}, 200$-time Monte Carlo approach is adopted for simulations. The simulation of the maneuvering midcourse warhead model is shown in Fig 3, we can tell that the 
influence in the ascent state of the missile is larger than that in the descent state. The maneuvering of the missile warhead influent the land position and the midcourse flight time will increase as well. The velocity of both the maneuvering missile warhead in the ascent state and the descent state enhanced when the missile midcourse flight ends, which may also let other problems like tracking loss or estimate errors in the boost phase tracking.

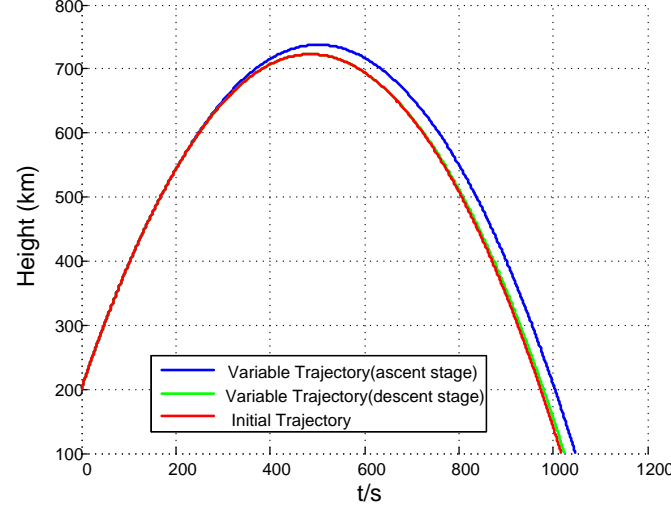

(a)Height Curve

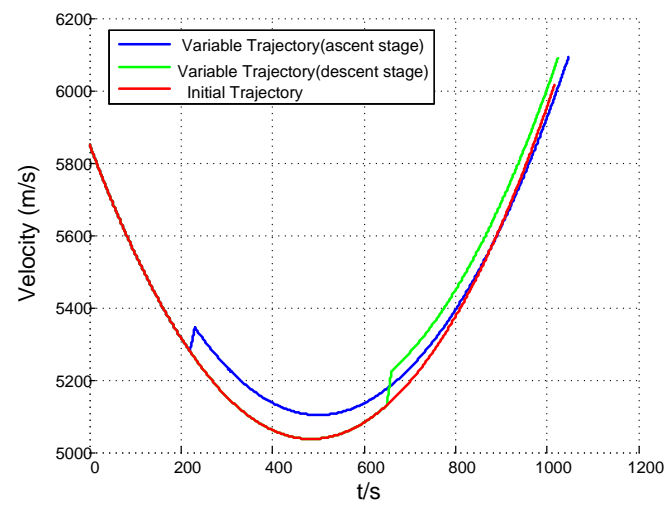

(b) Velocity Curve

Fig 3. Ballistic Trajectory characters comparison

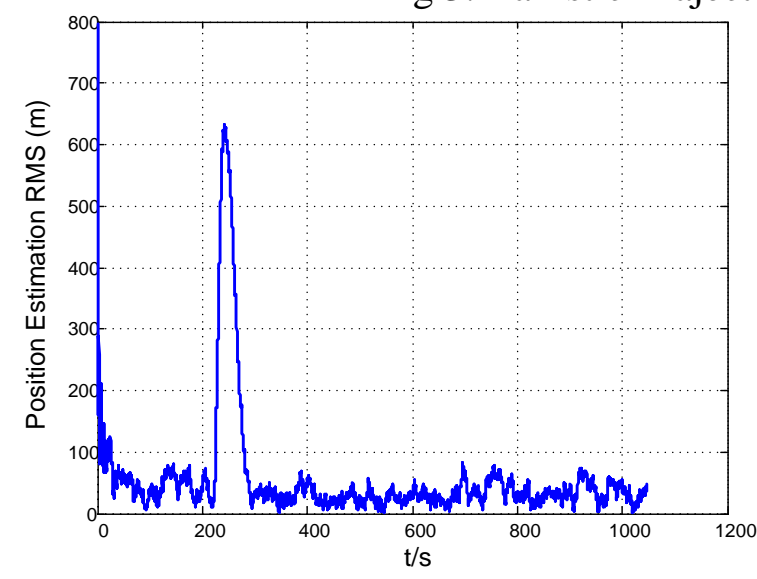

(a) Position Estimation RMSE

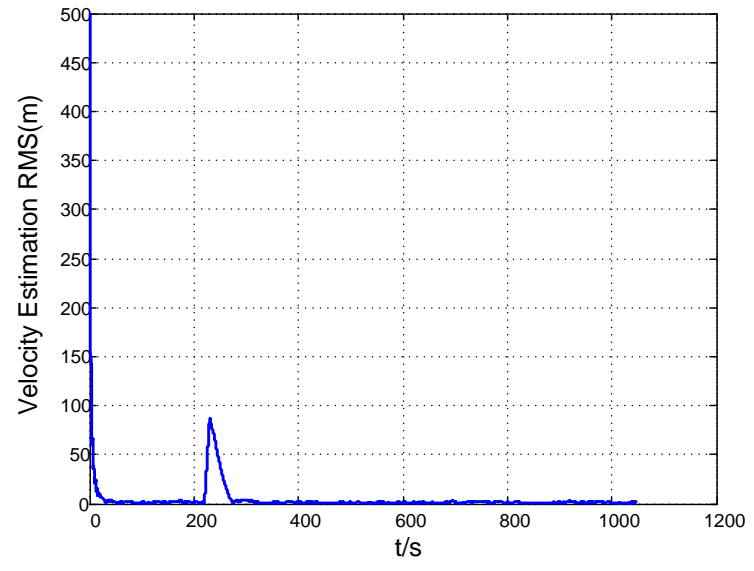

(b) Velocity Estimation RMSE

Fig 4.Characters of the maneuvering model in ascent stage

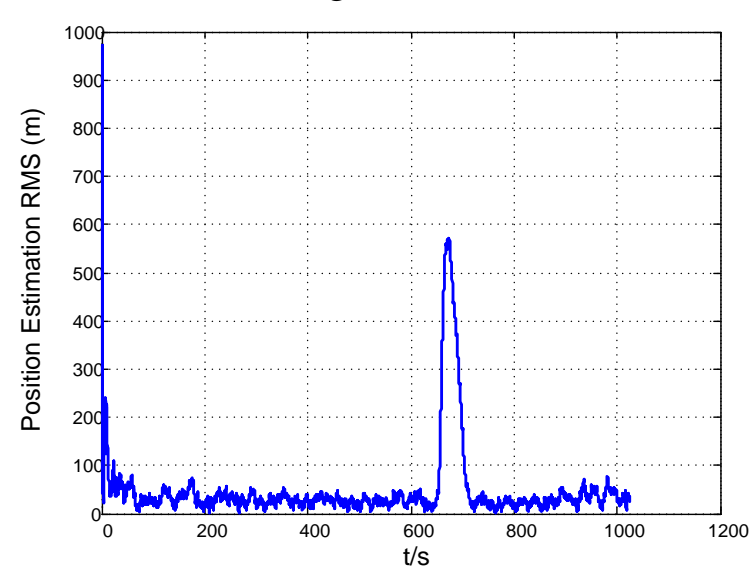

(a) Position Estimation RMSE

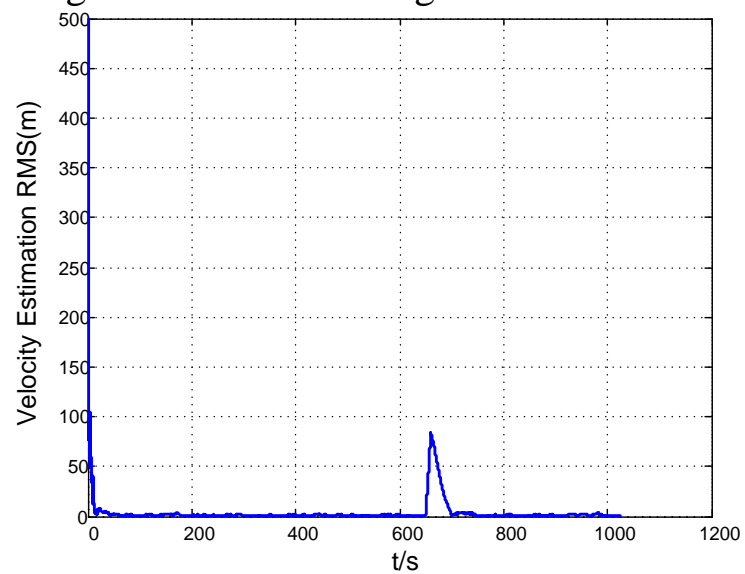

(b) Velocity Estimation RMSE

Fig 5.Characters of the maneuvering model in descent stage

In order to test the performance of the CKF, the EKF and UKF are used to compare with it. First the midcourse maneuvering model of ballistic missile is established. There are two types of midcourse maneuvering model, one's trajectory changes in the ascent stage and the other one change in the descent stage. The two kinds of midcourse maneuvering model are shown in Fig.3, which are calculated by the Runge-Kutta and compared with the non-maneuver cause. It can be seen that the performance of the CKF filter was demonstrated based on the Root Mean Square Error (RMSE) in 
target position and velocity. The position error curves were estimated by averaging 200 Monte-Carlo runs in Fig 4 and Fig 5. Fig 4 indicates that when the acceleration change of the ballistic missile. In Fig.6 and Fig.7, we have plotted the filtered estimates produced by the three filters, which indicates that when the acceleration change of the ballistic missile is caused by the thrust from the maneuvering warhead, CKF outperforms EKF and UKF. It can be seen that the estimates produced by CKF and UKF are close to each other and much closer to the true trajectory than that of EKF.

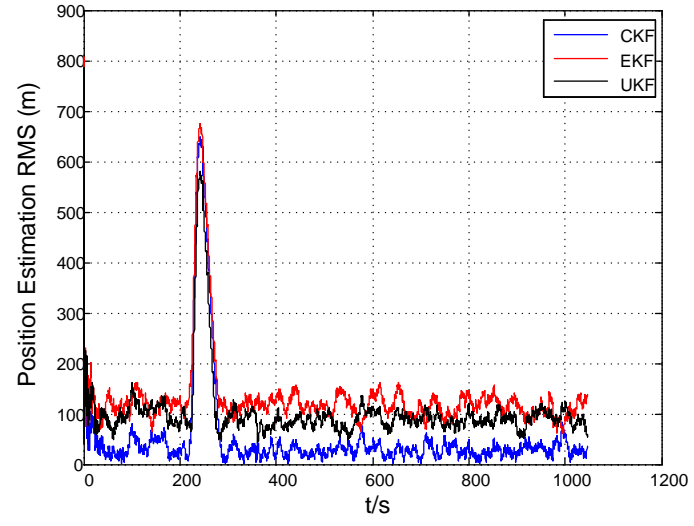

(a) Position Estimation RMSE

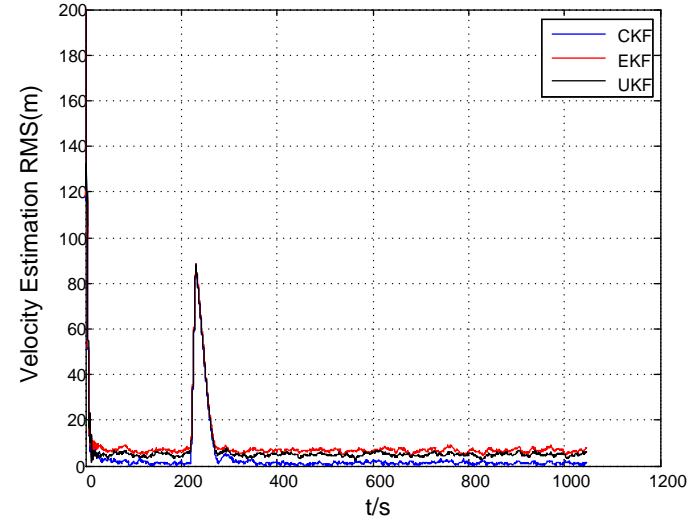

(b) Velocity Estimation RMSE

Fig 6.RMSEs of CKF, EKF and UKF in ascent stage

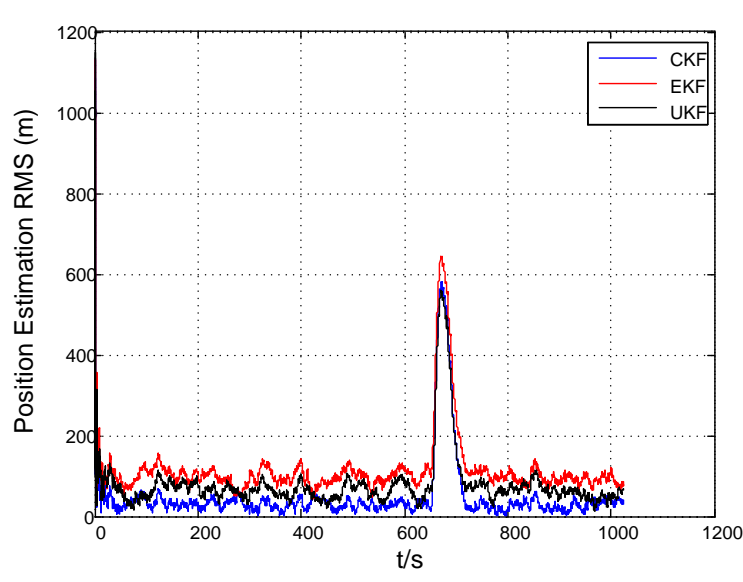

(a) Position Estimation RMSE

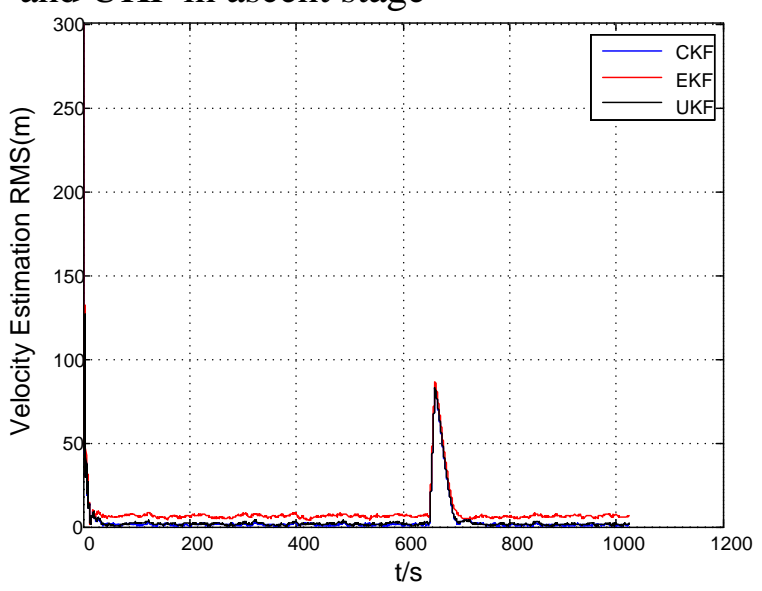

(b) Velocity Estimation RMSE

Fig.7 RMSEs of CKF, EKF and UKF in descent stage

The comparison is incomplete without mentioning the execution time of the three filters. The average central processing unit time is used to show as the execution time, which is displayed in table .1: The simulations show that CKF is more time-consuming than UKF. Considering 200-time Monte Carlo simulations, the time consumed by CKF is about 2.01 times as that by UKF. If the measurement equation is strong-nonlinear, this number will increase.

Table. 1 Average execution time of CKF, EKF, UKF

\begin{tabular}{c|ccc}
\hline Filters & CKF & EKF & UKF \\
\hline Average time(s) & 0.974 & 0.452 & 1.365 \\
\hline
\end{tabular}

\section{Conclusion}

With the development of the ballistic missile defense penetration technology, the midcourse ballistic missile can make maneuverable trajectory-change and there are abrupt changes in acceleration of target. In this paper, maneuvering midcourse ballistic warhead motion and measurement models are is studied, and the characteristics of cubature Kalman filter are analyzed respectively. The simulation results show that provides better estimation accuracy with minimal computational effort. Three filters such as EKF, UKF and CKF are compared for their performance and computational complexity. Simulation results show that the CKF performs the best in reducing the estimate errors, while the EKF is efficient approach for maneuvering target tracking with the 
worst performance. If the target is maneuverable and its acceleration has abrupt changes, CKF can lower the filtering error evidently to achieve an ideal performance with good precision and in less time.

\section{References}

[1]. Xin Xie, Weimin Li, Xiaoguang Zhou, Based Boost Phase Missile Tracking EKF Algorithm Modeling and Simulation. Modern defense technology, Vol.39,No.5, 2011, pp.146-151.

[2]. Branko Ristic, Sanjeev Arulampalam, Neil Gordon.Beyond the Kalman Filter: Partical Filters for Tracking Applications, Boston London: Artech House,2004,pp. 85-100.

[3]. Liu Yu, Wang Hong. UKF Based Nonlinear Filtering Using Minimum Entropy Criterion. IEEE Transactions On Signal Processing, Vol. 61,No.20,October 15, 2013,pp.45-49

[4]. Wenling.L., Yingmin.J.,Consesus-Based Distributed Multiple Models UKF for Jump Markov Nomlinear System. IEEE Transactions on Automatic Control. Vol.57,No.1,2012,pp.227-233

[5]. Yue Guo, Xin-xue Liu ,A Study on IMM-UPF Tracking Ballistic missiles at Free-flight Phase in Mixed Coordinate System . Journal of Ballistics, Vol.27,No.1,2015,pp.12-17.

[6]. Hui-ying Dong, Bin Cao, Yue-ping Yang. Application of Particle Filter for Target Tracking in Wireless Sensor Networks.International Conference on Communications and Mobile Computing, 2010,pp.504-508.

[7]. J. R. Katkuri, V. P. Jilkov, X. R. Li. A comparative study of nonlinear filters for target tracking in mixed coordinates.42nd South Eastern Symposium on System Theory University of Texas at Tyler, USA, 2010.

[8]. Yi-ou Sun, Jing-Wen Xie, Jun-Hai Guo. A novel CKF method for target tracking.IEEE,2014,pp.60-63.

[9]. Pei H. Leong,Sanjeev Arulampalam,Tharaka A. Lamahewa.A Gaussian-Sum Based Cubature Kalman Filter for Bearings-Only Tracking.IEEE Transactions on Aerospace and Electronic Systems, Vol.43,No.2,2013,pp.1161-1176.

[10]. Jinwhan Kim, P. K.Menon, Ernest Ohlmeyer. Motion Models for Use with the Maneuvering Ballistic Missile Tracking Estimators. AIAA Guidance, Navigation, and Control Conference, Vol. 7589, 2010.

[11]. Abhinoy Kumar Singhl, Shovan Bhaumikl. Cubature Quadrature Kalman Filter for Maneuvering Target Tracking .IEEE,2015,pp.24-29.

[12]. X. Rong Li, Vesselin, P. Jilkov. Survey of Maneuvering Target Tracking, Part II :Motion Models of Ballistic and Space Targets, IEEE Transactions on Aerospace and Electronic Systems vol. 46, No. 1 January 2010, 97-119.

[13]. Feng Zhang, Kang-Sheng Tian, Mu-Lin Xi. The Ballistic Missile Tracking Method Using Dynamic Model. Proceedings of 2011 IEEE CIE International Conference on Radar,2011,pp.26-29. 